\title{
Principles of Visualization in Radiation Oncology
}

\author{
Matthias Schlachter ${ }^{a}$ Bernhard Preim ${ }^{b, c}$ Katja Bühler ${ }^{a}$ Renata G. Raidou $^{d}$ \\ ${ }^{a}$ VRVis Research Center, Vienna, Austria; ${ }^{b}$ University of Magdeburg, Magdeburg, Germany; ${ }^{\mathrm{c}}$ Research Campus \\ STIMULATE, Magdeburg, Germany; ${ }^{\mathrm{d}}$ TU Wien, Vienna, Austria
}

\section{Keywords}

Medical visualization · Slice-based representations - Volume rendering $\cdot$ Multivolume visualization $\cdot$ Visual analytics

\begin{abstract}
Background: Medical visualization employs elements from computer graphics to create meaningful, interactive visual representations of medical data, and it has become an influential field of research for many advanced applications like radiation oncology, among others. Visual representations employ the user's cognitive capabilities to support and accelerate diagnostic, planning, and quality assurance workflows based on involved patient data. Summary: This article discusses the basic underlying principles of visualization in the application domain of radiation oncology. The main visualization strategies, such as slice-based representations and surface and volume rendering are presented. Interaction topics, i.e., the combination of visualization and automated analysis methods, are also discussed. Key Messages: Slice-based representations are a common approach in radiation oncology, while volume visualization also has a longstanding history in the field. Perception within both representations can benefit further from advanced approaches, such as image fusion and multivolume or hybrid rendering. While traditional slice-based and volume representations
\end{abstract}

keep evolving, the dimensionality and complexity of medical data are also increasing. To address this, visual analytics strategies are valuable, particularly for cohort or uncertainty visualization. Interactive visual analytics approaches represent a new opportunity to integrate knowledgeable experts and their cognitive abilities in exploratory processes which cannot be conducted by solely automatized methods.

(C) 2020 S. Karger AG, Base

\section{Introduction}

Visualization is the field of computer science that combines computer graphics elements to create visual data representations that make use of human vision and cognition [1]. Visual representations often support human interaction, to enhance the user's cognitive capabilities [2]. Visualization is used for data exploration to generate new knowledge [3,4], data analysis to verify hypotheses [5], and data presentation to communicate and externalize knowledge [6].

Visualization is used in many different application domains, including the medical field [6]. This use of technology is referred to as "medical visualization". Medical visualization emerged a couple of decades ago and became an influential field of research for many advanced karger@karger.com

www.karger.com/ocl

Karger!
(C) 2020 S. Karger AG, Basel

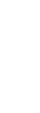

\footnotetext{
Matthias Schlachter

Biomedical Image Informatics Group

VRVis Research Center

Donau-City-Strasse 11, AT-1220 Wien (Austria)

E-Mail matthias.schlachter@vrvis.at
} 


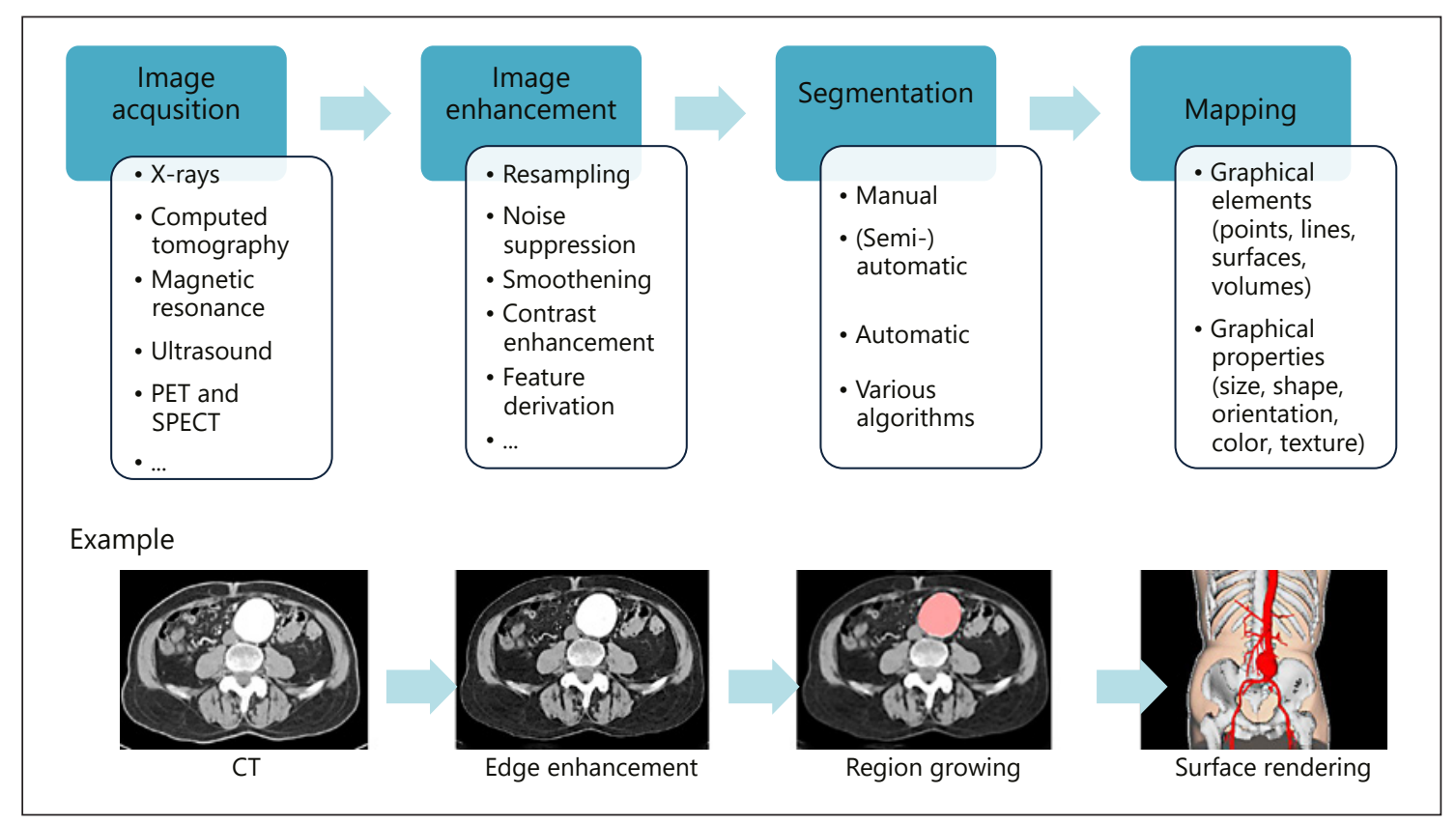

Fig. 1. The main steps of the medical visualization pipeline (image acquisition, enhancement, segmentation, and mapping) and a simple example involving aortic aneurysm visualization.

applications, including radiation oncology. The aim of this review is to provide insight into the fundamental principles of medical visualization and exemplify their typical application in radiation oncology.

Medical visualization can be classified as follows:

- Scientific visualization involves data with geometric structure or inherent spatial information, typically related to spatial data [7]. In medical visualization, these spatial data are medical imaging data, such as computed tomography (CT) and magnetic resonance imaging (MRI), or other data sources, such as radiotherapy treatment plans [8].

- Information visualization deals with abstract, nonphysical data [7]. In medical visualization, such data are, for example, retrieved from medical health records [9].

- Visual analytics [10] integrates concepts from the two previous categories with other disciplines, such as data mining, machine learning, or statistics. This is done in highly interactive environments that support data exploration and analysis $[4,11]$.

\section{The (Medical) Visualization Pipeline}

The visualization process consists of several steps [1]. These are known as the "visualization pipeline" and are presented in Figure 1. Visualization of medical image data follows the common visualization pipeline but is tuned to medical data and domain-specific requirements.

Initially, raw data are collected. In the medical domain, this happens during the imaging acquisition phase. The patient is scanned, and anatomical, pathological, and/or functional information is gathered in a stack of individual images of a specific modality, such as CT or MRI. Each image of a volume dataset represents a thin slice of the scanned patient and consists of elements, called pixels, arranged on a two-dimensional (2D) grid. These images are then reconstructed into volumes by various methods [6]. The reconstructed volumetric dataset combines $2 \mathrm{D}$ images into a $3 \mathrm{D}$ grid and consists of elements, called voxels. This configuration is shown in Figure $2 \mathrm{~b}$. Since a volume dataset is defined only at discrete grid positions, in-between samples are accessed by interpolation [12].

Image enhancement is then conducted to prepare the data for visualization [12]. This involves noise suppression, smoothening, and contrast enhancement methods. Segmentation is often performed on medical images to derive meaningful anatomical or pathological structures that can be reliably rendered. Multiple manual, automatic, or semiautomatic segmentation algorithms exist and are implemented depending on the data and application $[6,12]$. 
Fig. 2. a The main steps of the volume rendering process. $\mathbf{b}$ Schematic overview of arbitrary slices can be extracted from 3D volumes and how volumes are defined by a stack of slices commonly acquired by an imaging device. c Schematic overview of the ray casting algorithm and volume fusion. The ray is sampled at discrete positions to evaluate the volume-rendering integral.

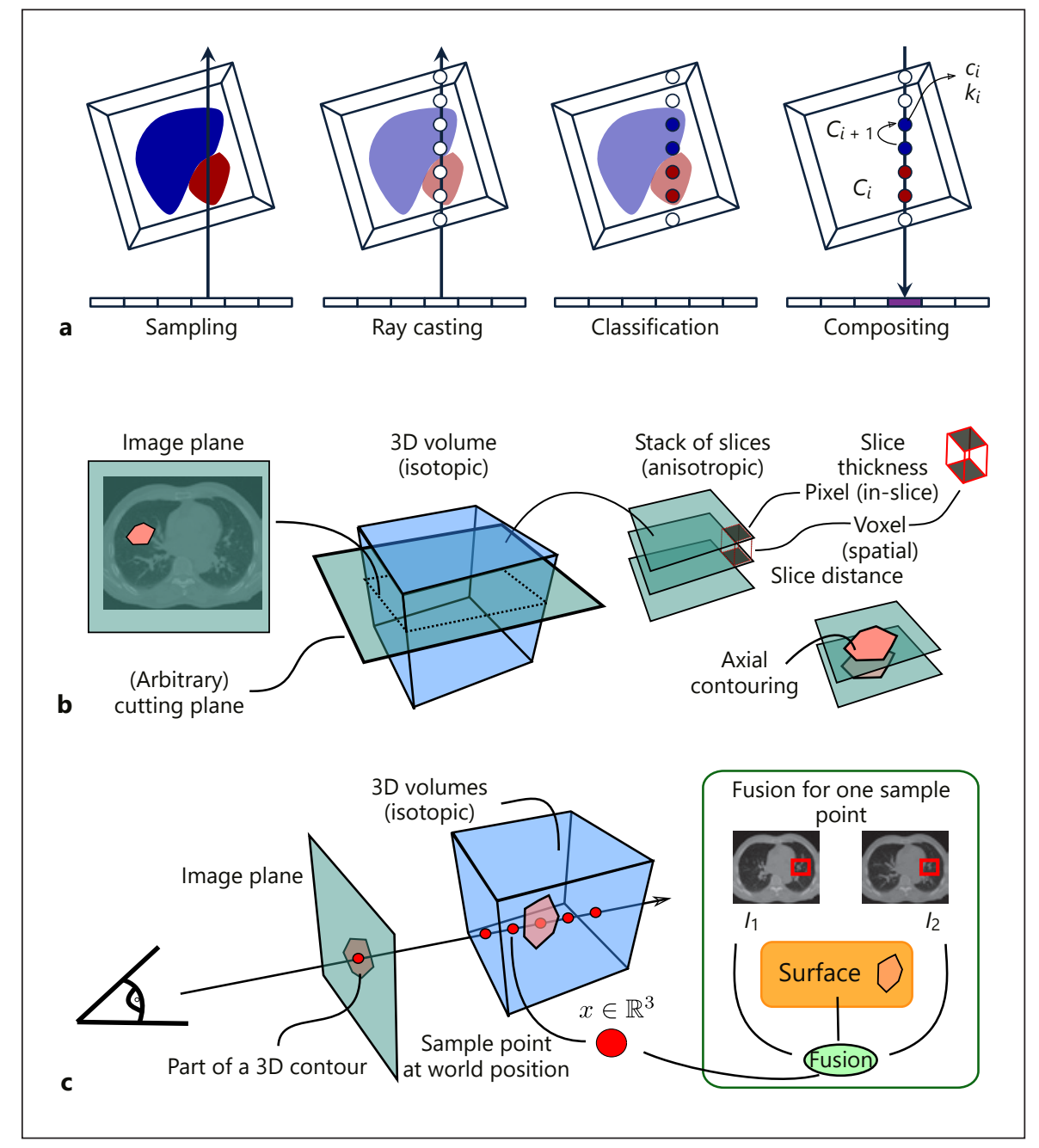

The enhanced and/or segmented data are subsequently mapped to graphical elements, i.e., points, lines, surfaces, or volumes, employing their graphical properties, i.e., size, orientation, color, texture, or shape [6]. The main techniques to visualize medical data on a screen are the following:

Slice-based representations include 2D slice-by-slice views with which the user can easily interact, as seen in Figure 3a. The slices often represent the three main anatomical planes. These slices are rendered in grayscale, and sometimes additional features are overlaid employing color and/or transparency.

Surface representations, surface rendering, or indirect volume rendering require the prior segmentation of structures, as seen in Figure $3 \mathrm{~b}$ (left). For example, this can be the result of a manual delineation or an automatic segmentation algorithm. After segmentation, the structure contours are mapped to polygonal surfaces, and dis- tinct colors and transparencies are assigned to each structure before rendering.

Volume representations, volume rendering, or direct volume rendering (DVR) require the direct projection of the entire data volume on the screen. Volume rendering is computationally intensive and consists of four steps, depicted in Figure 2a. For each pixel of the final image on the screen, a ray of sight is shot through the volume (ray casting) and samples are selected on positions along the ray (sampling). At these positions, materials, e.g., different kinds of tissue, are determined, and colors and transparencies are assigned to them (classification). Finally, all sample values along the ray are accumulated and numerically approximated to be represented on the screen (compositing).

Hybrid and multivolume representations are combinations of the above three categories, as depicted in Figure $3 b$. 


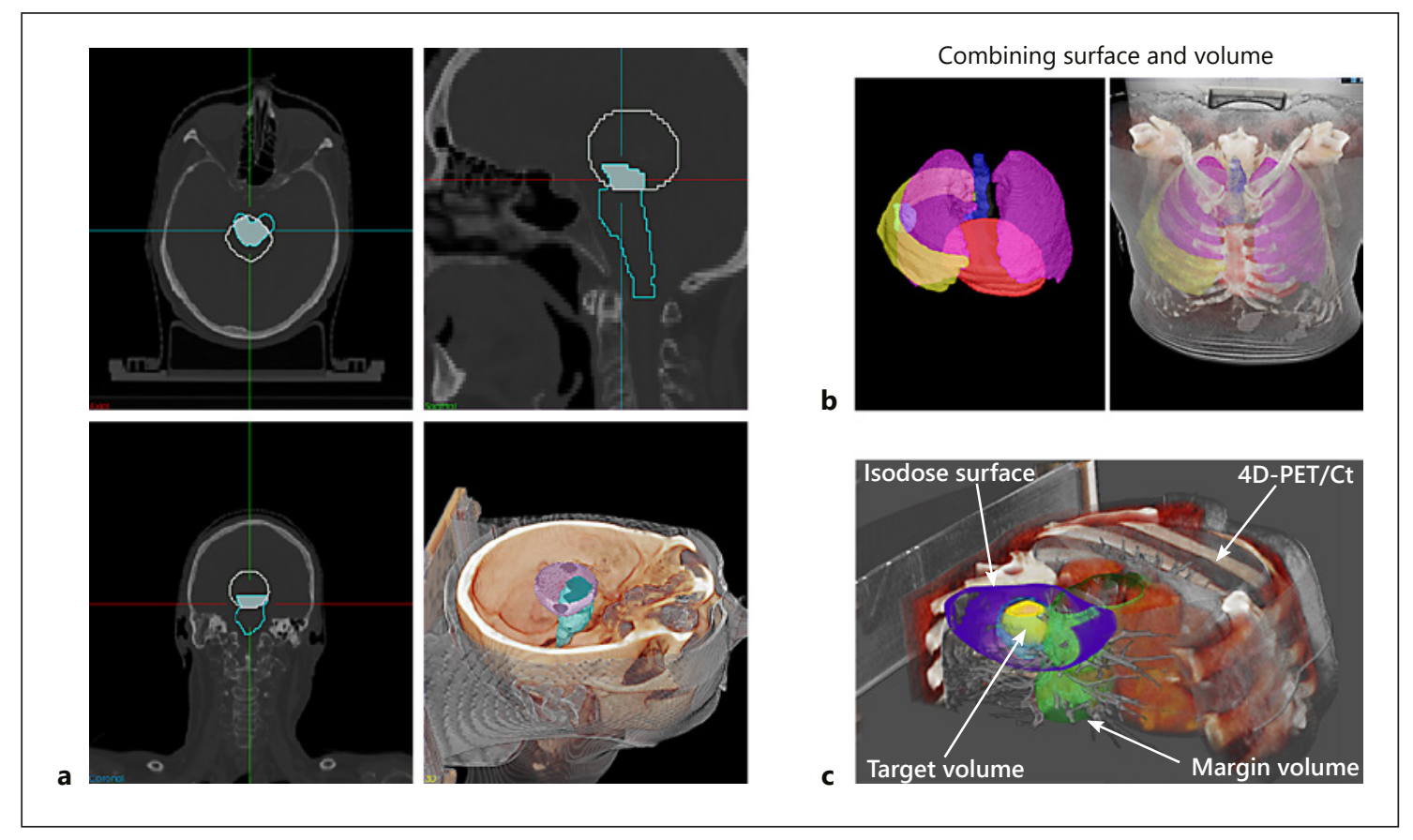

Fig. 3. a MPR views together with volume rendering of a head CT with segmentation data. b Surface representation of segmentation data (left) combined with CT data (right). c 4D-PET/CT combined with segmentation data and an isodose surface.

\section{Visualization Principles in Radiation Oncology}

In this section, we indicate the most significant trends within medical visualization research that are applied to radiation oncology. The goal of this review is not to present a comprehensive state-of-the-art report, as this has been already performed by Schlachter et al. [13]. Instead, we discuss selected principles of visualization in radiation oncology, in particular scientific visualization and visual analytics.

\section{Scientific Visualization for Radiation Oncology}

Volume visualization helps the understanding of 3D anatomical spatial relationships [14]. For example, in radiotherapy planning, it aids in the understanding of the relationship between anatomy and the radiation dose distribution. Imaging devices, such as CT scanners, usually create several projections of the human body which first need to be reconstructed into $2 \mathrm{D}$ slices to obtain the volumetric dataset. Reconstruction methods, such as filtered back-projection [6], are used to create a stack of slices, which are shown in Figure 2b. This is done in the following way. Assume a reconstructed CT dataset with a 1.17 $\times 1.17 \times 2 \mathrm{~mm}^{3}$ resolution. This resolution is anisotropic and can be problematic for image and, later, volume display and interpretation. To account for anisotropy, transforming the sample position and interpolating on the stack of slices is common in ray traversal, as depicted in Figure 2c. Alternatively, resampling the slices is also possible, but might lead to a loss of information or the addition of unnecessary data values. After this step, the data are ready for $2 \mathrm{D}$ and/or $3 \mathrm{D}$ visualization.

\section{D Representations}

In $2 \mathrm{D}$ representations, a standard slice-based approach is multiplanar reformation (MPR), a technique that extracts 2D slices from a 3D volume [15]. Although slices can be extracted in arbitrary orientations, a common way is to show three orthogonal planes, i.e., axial, coronal and sagittal, as shown in Figure 3a. Slice-based representations show a section of a volume defined by the intersection with a plane, as depicted in Figure $2 \mathrm{~b}$. The image information of the volume, e.g., an axial slice, is "reformatted" onto the plane and then displayed in the respective window. During reformatting, slice distance and inslice resolution are considered, to assure physically correct data display. 


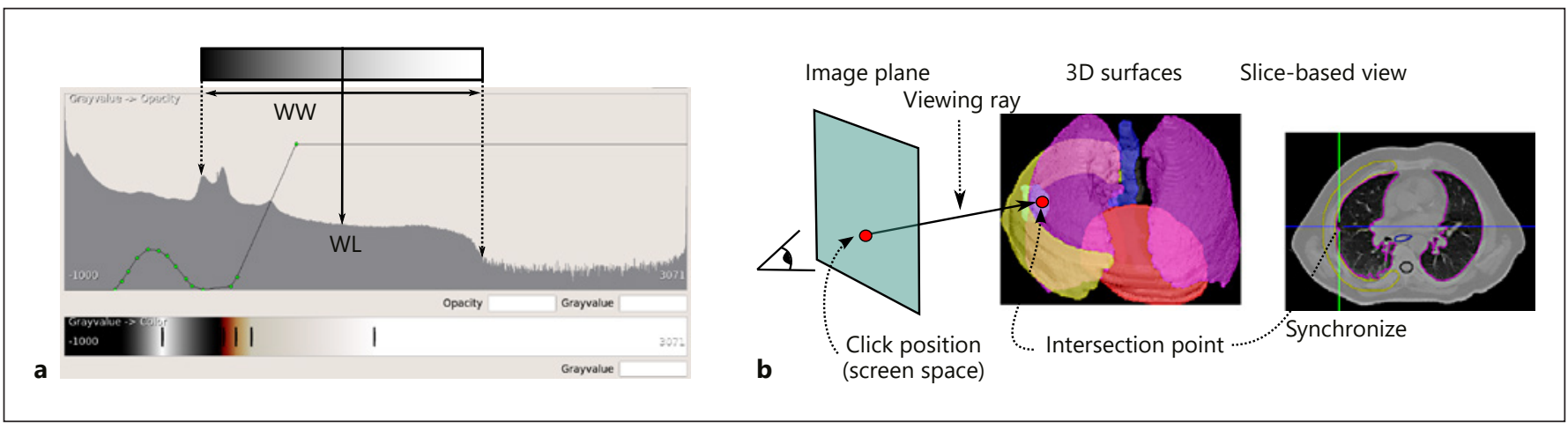

Fig. 4. a A widget used for defining a $1 \mathrm{D}$ transfer function. The upper part shows the histogram of a CT volume and points can be added to define the opacity transfer function, and the lower part to define the color transfer function, respectively. The concept of window/level is shown on top of the widget. Values outside the window are either black (to the left) or white (to the right). b Schematic overview of volume picking using a viewing sent from a screen position to determine the intersection with the depicted surfaces and synchronize a slice-based view.
Another important aspect is window/level. The basic concept of window/level is to apply a linear grayscale transform function specified by two parameters: window width (WW) and window level or window center (WL) to define how a subset of the entire dynamic range of the underlying data will be mapped to pixel intensities in the display. The WW defines the (relative) range of values, e.g., a Hounsfield unit range for $\mathrm{CT}$, and the $\mathrm{WL}$ is the midpoint of this range. When mapped to gray-level values (a pixel intensity of $0-255$ on an 8-bit display), values smaller or equal to $\mathrm{WL}-(\mathrm{WW} / 2)$ are black $(0)$, values greater or equal to $\mathrm{WL}+(\mathrm{WW} / 2)$ are white (255), and values in-between are mapped onto their corresponding pixel intensity. This is depicted in Figure 4a.

\section{D Representations}

We distinguish two general approaches for projecting $3 \mathrm{D}$ volume data onto the screen plane: DVR and surface rendering, also called indirect volume rendering. The latter usually involves image processing techniques, such as segmentation, to obtain triangulated surface meshes of distinct data structures [6]. Image enhancement and segmentation are beyond the scope of this paper. Please refer to the book by Gonzalez and Woods [12] for further information.

Direct Volume Rendering. DVR creates an interactive visualization of a volume without any intermediate preprocessing step. The method commonly assumes a simplified, physically motivated absorption/emission model of light propagation [16]. For DVR, multiple approaches exist, such as splatting, shear-warp, and texture mapping $[15,17]$. One of the most prominent and flexible DVR techniques is ray casting [17], especially since the existence of acceleration techniques using graphics hardware [18]. The idea behind ray casting is to cast a ray for each pixel of the screen from the origin of the camera through the volume. The rendering integral [17] is directly evaluated along the rays traversing the volume delivering the final pixel values. A schematic overview for one light ray is depicted in Figure 2a, c. The book by Engel et al. [17] offers a more detailed description.

Alpha blending [16] is a popular optical-blending technique, often implemented by using the Riemann sum to discretize the continuous function of the volume-rendering integral. Each sample in the approximation is assigned a color and opacity value. The Riemann sum when blending front-to-back for the current sample $i$ is then given by:

$$
C_{i+1}=C_{i}+\left(c_{i} \times k_{i}\right) \times\left(1-K_{i}\right) \text { and } K_{i+1}=K_{i}+k_{i} \times\left(1-K_{i}\right),
$$

where $c_{i}$ is the color and $k_{i}$ is the opacity of the current sample, and $C_{i}$ and $K_{i}$ are the accumulated values. The iteration schema for the composition of the color is depicted in Figure 2a (right).

Other simpler projection and compositing techniques exist, such as X-ray projection, and maximum intensity projection (MIP) [15]. When using MIP (Fig. 2c), only the value with highest intensity would be considered along the ray, but all other sample values would not contribute.

Transfer Functions. Volumetric data commonly consist of scalar values that represent a physical property, e.g., Hounsfield units denote radiodensity in CT data. In DVR, physical properties are assigned to optical properties, i.e., 


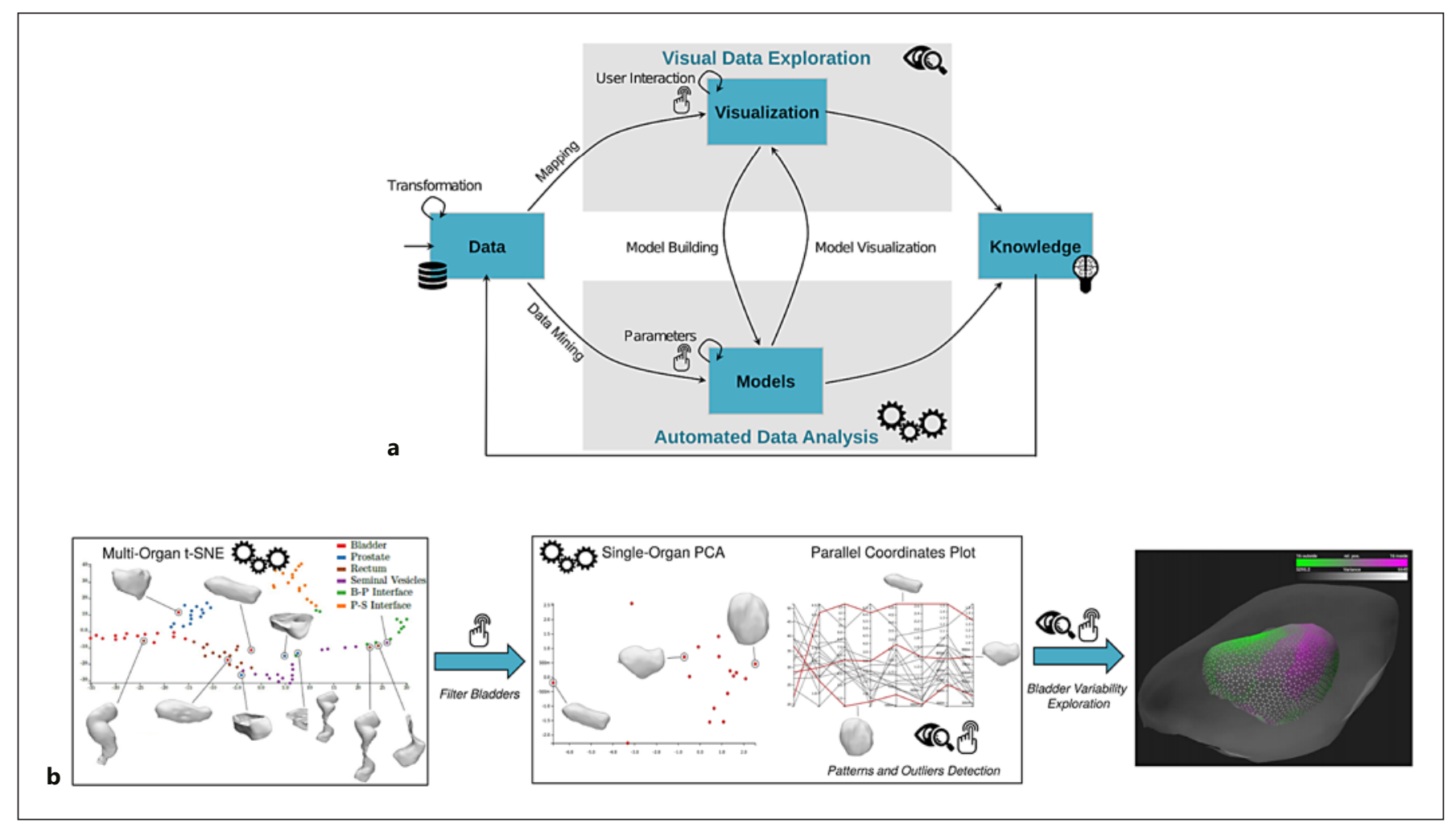

Fig. 5. a The main components of the visual analytics process. b An example of a tool for the exploration and analysis of the variability of pelvic organs [5].

color and transparency, as shown in Figure 3. Here, bones are displayed as opaque and skin tissue as more transparent. To obtain this, when the ray "hits" a bone, the accumulated opacity $K_{i}$ reaches a maximum value (opaque), i.e., no further light is visible from "behind" this point.

A widely used approach for assigning optical properties to the data values is by means of transfer functions in a process called classification [17], as shown in Figure 2a. When using a transfer function, each scalar value is assigned a color and opacity analogous to the window/level in slice-based representations. A common choice for color and opacity representation is RGBA, where the color is represented as combinations of red $(R)$, green $(G)$, and blue (B) channels, together with the opacity or alpha channel (A). The color transfer function $c: \mathbb{R} \rightarrow \mathbb{R}^{3}$ and opacity transfer function $k: \mathbb{R} \rightarrow \mathbb{R}$ are user-defined and can be defined graphically as depicted in Figure 4a. More complex approaches involve multidimensional transfer functions, e.g., those found in the survey by Ljung et al. [19].

Surface Representations. Delineations stemming from manual contouring, e.g., on axial slices (Fig. 2b), or binary volumes from segmentation algorithms are usually transformed into 3D meshes before rendering. For instance, delineations of target volumes in radiotherapy planning are commonly represented as surfaces, as well as isodose surfaces defined by Gy values. Surfaces can be visualized as opaque or with transparency, the latter enabling a better overview and understanding of the location of inner surfaces within outer surfaces [6]. For the correct rendering, all geometry primitives must be depth-sorted to be displayed in the correct spatial order, e.g., from back to front, to allow for a correct blending. In Figure $3 b$ (left) surface rendering of multiple, nested, and transparent contours of a lung cancer patient are visualized. Figure $3 \mathrm{c}$ shows, additionally, the isodose surfaces. In the corresponding MPR views, the segmentations (or isodoses) are often visualized as the outline of an area or as a filled (transparent) area overlaid on the slices (Fig. 3a). Here, the filled regions are used to highlight the overlap of two delineations. More information on surface rendering can be found in the books by Engel et al. [17] and Preim and Botha [6], where concepts for volume data in general are also discussed, such as shaded surface display for CT. 
Hybrid and Multivolume Representations

Hybrid Representations. Combining volume with surface rendering is essential in radiation oncology, to show, for example, combined isodose surfaces, delineations, and volumes in the same view (Fig. 3c). Technically, combining surfaces, as depicted in Figure 3b (left), with volume rendering (right) is quite challenging. In the example of ray casting, the algorithm needs to include the surface at the correct depth, as schematically depicted in Figure $2 c$, during the evaluation of the ray samples. This usually involves more advanced techniques and data structuring to correctly visualize these complex scenarios [20]. Realization for MPR views is simpler, as graphics programming languages, e.g., OpenGL [17], allow for directly overlaying image data with contour data, i.e., meshes or closed polygons.

Image Fusion and Multivolume Rendering. For multimodal visualization, processing is often required. When combining datasets from multiple modalities using separate scanners, registration needs to be performed to align the volumes. This is often costly in terms of processing time, so acceleration techniques may be necessary [21]. However, even when the transformation is known, different spatial resolutions, e.g., aligned PET/CT data with a 4 $\times 4 \times 4 \mathrm{~mm}^{3} / 1.17 \times 1.17 \times 2 \mathrm{~mm}^{3}$ resolution, add to the complexity. One way to implement fusion during ray casting is on the classification level [22], where the optical properties for a sample point of two or more volumes are combined (Fig. 2c). The fusion of the volume $I_{1}$ and $I_{2}$ is simply given by a weighted linear combination. Let $c_{1}$ and $c_{2}$ denote the color value of $I_{1}$ and $I_{2}$ at world position $x$ (Fig. 2c). Then the fusion of the color is given by $c_{f}=\alpha \times$ $c_{1}+(1-\alpha) \times c_{2}$, and can be defined for the absorption, respectively. The parameter $\alpha \in[0,1] \subset \mathbb{R}$ is user-defined and can be implemented, for instance, as a slider for changing the weight. This parameter can also be used for the slice-based visualization of the axial, sagittal, and coronal views, where the images are fused, respectively. The fusion for slice-based visualizations is usually easier to implement as graphics programming languages allow for directly blending (overlaying) whole images. A more detailed overview can be found in the surveys on medical image fusion by James and Dasarathy [23], on multimodal data visualization by Lawonn et al. [24], and on multivolume ray casting approaches by Lux and Fröhlich [25].

Volume Rendering Using Graphics Processing Unit. Most of the previously discussed algorithms can leverage modern graphics processing units (GPUs). For most of the algorithms GPU-based acceleration techniques for volume rendering exist [18]. A survey comparing differ- ent ray casting techniques using GPUs is provided by Schubert and Scholl [22].

\section{Interaction}

An integral part of medical visualization is interaction. One of the most common interaction tasks is to change visual parameters [6], e.g., change transfer functions by adding points to the widget shown in Figure $4 \mathrm{a}$, and therefore changing the optical properties assigned to the scalar values of a dataset as explained above. A common interaction for slice-based visualization is panning and zooming, to refine the visual area of interest by moving the screen or the view on the screen (pan) and zooming into the area of interest. Navigation through the volume along the cutting direction for MPR views is another example. Here, the visualizations must be aware of slice distances to correctly display and move the cutting plane, as shown in Figure $2 \mathrm{~b}$. For volume rendering, interaction techniques such as $3 \mathrm{D}$ picking (Fig. $4 \mathrm{~b}$ ), where the closest surface point along the view direction from the 2D mouse position can be selected and the $2 \mathrm{D}$ views will be rearranged to show the position of the surface point. More advanced interactions include cutting and clipping [26], used in Figure $3 c$ to "cut open" the volume, or volume masking, where certain regions can be assigned to a segmentation to further influence the volume fusion [8].

\section{Visual Analytics for Radiation Oncology}

Up to this point, we have discussed the visualization of medical data which have a 3D anatomical spatial arrangement, such as CT or MRI data and RT treatment plans. However, additional nonspatial data might be available, such as electronic health records of a patient or data from population studies. For these data, the methods discussed in the previous section are not sufficient. Moreover, given the steadily increasing dimensionality and complexity of medical data, new strategies have emerged as a natural response for visualization, exploration, and analysis. For instance, the need to integrate morphological with functional information has led to multimodal imaging [24]. Despite recent advances in data analysis techniques, the exploration of multimodal data using the methods in the previous section is still cumbersome. Interactive approaches represent a new opportunity to integrate knowledgeable experts and their cognitive abilities in the exploratory process.

According to Keim et al. [27]: "Visual analytics combines automated analysis techniques with interactive visualizations for effective understanding, reasoning and decision making on the basis of very large and complex 
datasets". The process and components of visual analytics are depicted in Figure 5a. Visual analytics makes use of the visualizations discussed above (The Visualization Pipeline), as well as other commonly employed visual representations from the domain of information visualization, such as scatterplots, scatterplot matrices, or parallel coordinates plots. Additionally, visualization is combined with other disciplines, most commonly statistics, data mining, or machine learning. A human-in-the-loop approach is essential in visual analytics solutions, integrating human strengths for sense and decision-making with semiautomated data analysis [2,7]. This is enabled by interaction. Visual analytics incorporates several fundamental techniques, methods and concepts:

- The visual analytics seeking mantra [10] is summarized as: Analyze first - Show the important - Zoom, filter and analyze further - Details- on-demand. Initially, processes for the reduction or abstraction of the data are employed, so that only the important data can be displayed. For example, data mining serves the purpose of removing outliers or of computing clusters or correlations. Then, users zoom in and filter the data for more detailed information. At the end, all detailed information is shown on demand through interaction.

- Multiple (coordinated) views [28] are widely used in visual analytics. This refers to employing multiple views that allow the observation of data and their inbetween relations from different perspectives. Multiple views are usually combined with brushing and linking (discussed below) to facilitate the identification of relationships within data.

- Brushing and linking [29] (B/L) is a concept that involves selecting one or several interesting data subsets in one view and highlighting corresponding subsets in another. This method is meant to overcome the shortcomings of single techniques, and it provides more information than the exploration of individual views.

- Focus + context $[1](\mathrm{F}+\mathrm{C})$ is required to present items at different levels of detail. More interesting or relevant data subsets are presented with more detail, while less important ones are presented with less detail. These are, however, retained in the view to provide the context for a better understanding and insight.

- Overview + detail [30] is related to the combined use of multiple views and $\mathrm{F}+\mathrm{C}$. Here, at least two views are presented to the user: one with a rough overview on the entire visualization space and one with a detailed view of a smaller portion of the space.

Visual analytics solutions tend to be powerful and complex. Often, target users are not able to fully exploit the potential of such systems. Guided visual analytics puts emphasis on the effective use of such systems by domain experts by integrating guidance concepts [31].

Due to the tight and interactive coupling of the components of visual analytics, it is preferable to present a few example applications focusing on two tasks, often encountered in radiation oncology. In this article, we focus on (i) radiotherapy-related approaches for the exploration of tissue characterization employed in the target volumes definition, and (ii) strategies for the analysis and assessment of organ segmentation outcomes.

In the first category, visual analytics for tissue characterization initially provided solutions, such as the interactive framework of Coto et al. [32] for the exploration and analysis of breast dynamic contrast-enhanced (DCE) MRI data. Here, 2D and 3D anatomic representations of patient data are used together with scatterplots representing the contrast agent enhancement. These are integrated through $\mathrm{B} / \mathrm{L}$, enabling the identification and characterization of breast lesions. In other solutions, the entire space of tissue characteristics is visualized after a dimensionality reduction step $[3,33,34]$.

In the second category, visual analytics approaches have been employed for the analysis and assessment of automatic segmentations, which rely heavily on the interaction concepts discussed above. Organ segmentations can be used, e.g., as input to radiotherapy treatment planning. Therefore, their accuracy is crucial. There are approaches that focus on supporting both cohort and individual patient investigation for the detailed assessment of organ segmentation accuracy [35]. Reiter et al. [4] investigated how the shape and size of organs affect the accuracy of automatic segmentation methods and enable quick identification of segmentation errors and their correlation to anatomical features, depicted in Figure 5b. Visual analytics within the domain of radiotherapy has been further investigated by Raidou et al. [11], tackling several steps of the treatment pipeline.

\section{Discussion}

Slice-based representations are still one of the most common (and maybe preferred) ways to visualize imaging data. This might be because contouring is often performed on axial slice views, making the adaptation to other visualizations difficult. Also, slices are easier to use as they require fewer visual parameters, e.g., transfer functions versus level/window. Additionally, in the presence of multiple volumes, contours and isodose surfaces, the views are 
often described as "cluttered", i.e., too much information is displayed at once. Advanced concepts such as smart visibility $[36,37]$ or cutaway views [38] are employed to show only the most relevant information. The former usually requires knowledge of the underlying data whereas the latter requires user interaction [24]. Still, enhancing slicebased representations is an ongoing task [39].

Volume visualization has a long history in radiation oncology. Visualizations employing region boundary surfaces to display anatomy, polygonal meshes to display treatment beams, and isodose surfaces to display dose were already presented 30 years ago [14]. Even though advanced volume rendering can, for instance, improve the assessment of contours in $4 \mathrm{D}$ imaging [8], simpler techniques, such as MIP might still be preferred, as they can suffice for a specific application [40]. Another important aspect of volume rendering is perception. When discussing delineation visualizations, illustrative techniques can be employed to greatly enhance depth perception [41]. Volumetric illumination can further improve perception [42].

While traditional slice-based and volume representations keep evolving, at the same time the dimensionality of medical data is "exploding". Patient cohort acquisitions are often employed in retrospective studies, and population studies are becoming widespread [43]. The data of these studies are complex and heterogeneous, but they offer valuable information for clinical research. For this kind of data, visual analytics can be particularly suitable. Early examples of visual analytics tools for the analysis of cohort or population data within the radiation oncology domain involve topics related to anatomical variability and its effect on therapy. Raidou et al. [5] targeted the exploration of the anatomical variability of the bladder and the analysis of potential toxicity risk for prostate cancer patients. Shape analysis is conducted using unfoldings of the involved organs on a plane, where several attributes can be color-encoded. Regarding chemotherapy optimization, ChemoExplorer supports the analysis of different treatment strategies and an understanding of how different groups of patients respond to selected therapies, combining visualizations of imaging and non-imaging health record data that facilitate the comparison of patient subgroups [9].

Moreover, uncertainty associated with medical data often needs to be addressed. Uncertainty is present in all kinds of medical data and processes and relates to different concepts, such as errors, imprecisions, subjectivity, sensitivity to (small) parameter changes, and non-specificity [44]. In medical visualization research, uncertainty has drawn more attention in the last few years [45]. Nguyen et al. [46] have proposed the use of an interactive ex- ploratory tool that supports uncertainty minimization in kinetic imaging, while iCoCooN [47] enables, in addition, the identification of relations between kinetic parameters and their variability. Both tools are equipped with interactive brushing that bidirectionally links the observations in the kinetic space to patient anatomy.

Finally, an important component of medical visualization is the assessment of the usefulness of the developed techniques and applications. Often, this is done with empirical evaluations involving participants from the target user group [48], but more concrete guidelines are required that incorporate both qualitative and quantitative methods. For example, as processing is often required, quantitatively evaluating the results of a visualization is important, e.g., in registration applications [49]. Algorithm verification, such as for the comparative evaluation of volume-rendering algorithms [50], is also required, to increase the level of trust in commonly employed visual data analysis strategies. There is, too, an increasing need for evaluations with respect to usability, functionality and user experience, in realistic settings [34], to obtain valid feedback on developed visualization tools.

\section{Conclusion}

We have summarized the basic underlying principles of volume visualization and visual analytics in the application domain of radiation oncology. Important topics, such as DVR and surface rendering of common data used in radiation oncology applications, as well as user interaction, have been presented. More advanced techniques, including multivolume rendering and perception-motivated visualizations, and topics such as the evaluation of visualization designs have been discussed. We anticipate that future challenges related to the complexity of data and processes of radiation oncology can benefit from the domain of visual analytics.

\section{Acknowledgement}

This paper was partly written in collaboration with the VRVis Competence Center. VRVis is funded by BMVIT, BMDW, Styria, SFG, and the Vienna Business Agency in the scope of COMET (Competence Centers for Excellent Technologies [854174]), which is managed by FFG.

\section{Author Contributions}

All coauthors contributed equally to writing this paper. 


\section{References}

1 Card SK, Mackinlay JD, Shneiderman B, editors. Readings in Information Visualization: Using Vision to Think. Burlington (MA): Morgan Kaufmann; 1999.

2 Munzner T, editor. Visualization Analysis and Design. Boca Raton (FL): CRC Press; 2014.

3 Nunes M, Rowland B, Schlachter M, Ken S, Matkovic K, Laprie A, et al. An Integrated Visual Analysis System for Fusing MR Spectroscopy and Multi-Modal Radiology Imaging. IEEE Conference on Visual Analytics Science and Technology; 2014. Available from: https://doi.org/10.1109/VAST.2014.7042481.

4 Reiter O, Breeuwer M, Gröller E, Raidou RG. Comparative Visual Analysis of Pelvic Organ Segmentations. EuroVis - Short Papers; 2018. pp. 37-41.

5 Raidou RG, Casares-Magaz O, Amirkhanov A, Moiseenko V, Muren LP, Einck JP, et al. Bladder Runner: Visual Analytics for the Exploration of RT-Induced Bladder Toxicity in a Cohort Study. Comput Graph Forum. 2018; 37(3):205-16.

6 Preim B, Botha CP. Visual Computing for Medicine: Theory, Algorithms, and Applications. Oxford: Newnes; 2013.

7 Tory M, Möller T. Human factors in visualization research. IEEE Trans Vis Comput Graph. 2004 Jan-Feb;10(1):72-84.

8 Schlachter M, Fechter T, Adebahr S, SchimekJasch T, Nestle U, Bühler K. Visualization of 4D multimodal imaging data and its applications in radiotherapy planning. J Appl Clin Med Phys. 2017 Nov;18(6):183-93.

9 Karall N, Gröller E, Raidou RG. ChemoExplorer: A Dashboard for the Visual Analysis of Chemotherapy Response in Breast Cancer Patients. EuroVis 2018 - Short Papers. The Eurographics Association; 2018. pp. 49-53.

10 Keim D, Andrienko G, Fekete JD, Görg C, Kohlhammer J, Melançon G, editors. Visual Analytics: Definition, Process, and Challenges. Information visualization. Springer; 2008. pp. 154-75.

11 Raidou RG, Breeuwer M, Vilanova A. Visual Analytics for Digital Radiotherapy: Towards a Comprehensible Pipeline. In: Eurographics - Dirk Bartz Prize; 2017. pp. 1-4.

12 Gonzalez RC, Woods RE, editors. Digital Image Processing. 2nd ed. Upper Saddle River (NJ): Prentice-Hall, Inc.; 2002

13 Schlachter M, Raidou RG, Muren LP, Preim B, Putora PM, Bühler K. State-of-the-Art Report: Visual Computing in Radiation Therapy Planning. EUROVIS 2019;38(3).

14 Levoy M, Fuchs H, Pizer SM, Rosenman J, Chaney EL, Sherouse GW, et al, editors. Volume Rendering in Radiation Treatment Planning. Proceedings of the 1st Conference on Visualization in Biomedical Computing; 1990 May; Atlanta (GA). https://doi.org/10.1109/ VBC.1990.109295.
15 Zhang Q, Eagleson R, Peters TM. Volume visualization: a technical overview with a focus on medical applications. J Digit Imaging. 2011 Aug;24(4):640-64.

16 Max N. Optical Models for Direct Volume Rendering. IEEE Trans Vis Comput Graph. 1995;1(2):99-108.

17 Engel K, Hadwiger M, Kniss JM, Rezk-Salama C, Weiskopf D. Real-time Volume Graphics. Natick (MA): AK Peters; 2006.

18 Krüger J, Westermann R. Acceleration Techniques for GPU-based Volume Rendering. Proceedings of the 14th IEEE Visualization 2003 (VIS'03); Washington DC. IEEE Computer Society; 2003. pp. 287-92.

19 Ljung P, Krüger J, Gröller E, Hadwiger M, Hansen CD, Ynnerman A. State of the Art in Transfer Functions for Direct Volume Rendering. Comput Graph Forum. 2016 Jun; 35(3):669-91

20 Kainz B, Grabner M, Bornik A, Hauswiesner S, Muehl J, Schmalstieg D. Ray Casting of Multiple Volumetric Datasets with Polyhedral Boundaries on Manycore GPUs. Proceedings of Siggraph ASIAA; 2009; Yokohama. https://doi.org/10.1145/1661412.1618498.

21 Fluck O, Vetter C, Wein W, Kamen A, Preim B, Westermann R. A survey of medical image registration on graphics hardware. Comput Methods Programs Biomed. 2011 Dec; 104(3):e45-57.

22 Schubert N, Scholl I. Comparing GPU-based Multi-Volume Ray Casting Techniques. Comput Sci Res Dev. 2011;26(1):39-50.

23 James AP, Dasarathy BV. Medical Image Fusion: A Survey of the State of the Art. Inf Fusion. 2014;19:4-19.

24 Lawonn K, Smit NN, Bühler K, Preim B. A Survey on Multimodal Medical Data Visualization. Comput Graph Forum. 2018;37(1): 413-38.

25 Lux C, Fröhlich B. GPU-based Ray Casting of Multiple Multi-Resolution Volume Datasets. Advances in Visual Computing; 2009. pp. 104-16.

26 Weiskopf D, Engel K, Ertl T. Volume Clipping via Per-Fragment Operations in Texture-Based Volume Visualization. Proceedings of IEEE Visualization 2002. IEEE Computer Society; 2002. pp. 93-100.

27 Keim DA, Kohlhammer J, Ellis G, Mansmann F. Mastering the Information Age-Solving Problems with Visual Analytics. Proceedings of Eurographics; 2010.

28 Wang Baldonado MQ, Woodruff A, Kuchinsky A. Guidelines for Using Multiple Views in Information Visualization. Advanced Visual Interfaces. ACM; 2000. pp. 110-9.

29 Becker RA, Cleveland WS. Brushing Scatterplots. Technometrics. 1987;29(2):127-42.

30 Cockburn A, Karlson A, Bederson BB. A Review of Overview+Detail, Zooming, and Focus+Context Interfaces [CSUR]. ACM Comput Surv. 2009;41(1):2
31 Ceneda D, Gschwandtner T, May T, Miksch S, Schulz HJ, Streit M, et al. Characterizing Guidance in Visual Analytics. IEEE Trans Vis Comput Graph. 2017 Jan;23(1):111-20.

32 Coto E, Grimm S, Bruckner S, Gröller ME, Kanitsar A, Rodriguez O, editors. MammoExplorer: An Advanced CAD Application for Breast DCE-MRI. Proceedings of Vision, Modelling, and Visualization; 2005; Erlangen. pp. 91-8.

33 Oeltze S, Doleisch H, Hauser H, Muigg P, Preim B. Interactive visual analysis of perfusion data. IEEE Trans Vis Comput Graph. 2007 Nov-Dec;13(6):1392-9.

34 Raidou RG, van der Heide UA, Dinh CV, Ghobadi G, Kallehauge JF, Breeuwer M, et al. Visual Analytics for the Exploration of Tumor Tissue Characterization. Comput Graph Forum. 2015 Jun;34(3):11-20.

35 Raidou RG, Marcelis FJ, Breeuwer M, Gröller E, Vilanova A, van de Wetering HM. Visual Analytics for the Exploration and Assessment of Segmentation Errors. Proceedings of Eurographics Workshop on Visual Computing for Biology and Medicine; 2016. pp. 193-202.

36 Jung Y, Kim J, Eberl S, Fulham M, Feng DD. Visibility-driven PET-CT Visualisation with Region of Interest (ROI) Segmentation. Vis Comput. 2013;29(6-8):805-15.

37 Viola I, Gröller E. Smart Visibility in Visualization. In: Neumann L, et al, editors. Computational Aesthetics in Graphics, Visualization and Imaging; 2005. Eurographics Assoc 2005. pp. 209-216.

38 Diepstraten J, Weiskopf D, Ertl T. Interactive Cutaway Illustrations. Comput Graph Forum. 2003 Sep;22(3):523-32.

39 Tietjen C, Meyer B, Schlechtweg S, Preim B, Hertel I, Strau G. Enhancing Slice-based Visualizations of Medical Volume Data. In: Eurographics/IEEE VGTC Conference on Visualization; 2006. pp. 123-130.

40 Fishman EK, Ney DR, Heath DG, Corl FM, Horton KM, Johnson PT. Volume rendering versus maximum intensity projection in CT angiography: what works best, when, and why. Radiographics. 2006 May-Jun;26(3): 905-22.

41 Tietjen C, Isenberg T, Preim B. Combining Silhouettes, Surface, and Volume Rendering for Surgery Education and Planning. In: Eurographics/IEEE VGTC Conference on Visualization; 2005. pp. 303-10.

42 Preim B, Baer A, Cunningham D, Isenberg T, Ropinski T. A Survey of Perceptually Motivated 3D Visualization of Medical Image Data. Comput Graph Forum. 2016;35(3): 501-25.

43 Preim B, Klemm P, Hauser H, Hegenscheid K, Oeltze S, Toennies K, et al. Visual Analytics of Image-Centric Cohort Studies in Epidemiology. Visualization in Medicine and Life Sciences III. Springer Int.; 2016. pp. 221-48. 
44 Raidou RG. Uncertainty Visualization: Recent Developments and Future Challenges in Prostate Cancer Radiotherapy Planning. In: EuroVis Workshop on Reproducibility, Verification, and Validation in Visualization; 2018. pp. 13-17.

45 Ristovski G, Preusser T, Hahn HK, Linsen L. Uncertainty in Medical Visualization: towards a Taxonomy. Comput Graph. 2014;39:60-73.

46 Nguyen KT, Bock A, Ynnerman A, Ropinski T. Deriving and Visualizing Uncertainty in Kinetic PET Modeling. In: Eurographics
Workshop on Visual Computing for Biology and Medicine; 2012. p. 107-114.

47 Raidou R, van der Heide UA, van Houdt P, Breeuwer M, Vilanova A. The iCoCooN: Integration of Cobweb Charts with Parallel Coordinates forVisual Analysis of DCE-MRI Modeling Variations. In: Eurographics Workshop on Visual Computing for Biology and Medicine; 2014. pp. 11-20.

48 Preim B, Ropinski T, Isenberg P. A Critical Analysis of the Evaluation Practice in Medical Visualization. In: Eurographics Workshop on
Visual Computing for Biology and Medicine; 2018. pp. 45-56.

49 Schlachter M, Fechter T, Jurisic M, SchimekJasch T, Oehlke O, Adebahr S, et al. Visualization of Deformable Image Registration Quality Using Local Image Dissimilarity. IEEE Trans Med Imaging. 2016 Oct;35(10):2319-28.

50 Etiene T, Jönsson D, Ropinski T, Scheidegger C, Comba JL, Nonato LG, et al. Verifying volume rendering using discretization error analysis. IEEE Trans Vis Comput Graph. 2014 Jan;20(1):140-54. 